\title{
Ivor Lewis minimally invasive esophagectomy for esophageal cancer: An excellent operation that improves with experience
}

\author{
Abby White, DO, ${ }^{a}$ Suden Kucukak, MD, ${ }^{a}$ Daniel N. Lee, BS, ${ }^{a}$ Emanuele Mazzola, PhD, ${ }^{b}$ \\ Yong Zhang, MD, ${ }^{\mathrm{c}}$ and Scott J. Swanson, $\mathrm{MD}^{\mathrm{a}}$
}

\section{ABSTRACT}

Objective: Esophagectomy is associated with major morbidity. In this study we sought to assess the learning curve of minimally invasive Ivor Lewis esophagectomy (MIILE) and to evaluate perioperative outcomes, including anastomotic leak and hospital readmission, as a function of graduated surgeon experience.

Methods: Data were extracted from the electronic medical records of patients who underwent MIILE, performed by a single surgeon over an 8-year period (2009-2017). Primary outcomes were 5-year overall survival, postoperative complications, and 90-day readmission rates. Surgeon experience was divided into 4 quartiles, representing graduated experience. Statistical analysis was performed using univariate and multivariate logistic regression, whereas Kaplan-Meier estimators were used to assess survival outcomes.

Results: A total of 170 patients underwent MIILE and were analyzed after exclusion criteria were applied. Five-year overall survival was $50.1 \%$ (95\% confidence interval, 39.7\%-63.2\%). Mortality at 90 days was $3.9 \%$ (95\% confidence interval, 0.8\%-6.9\%). Major complications occurred in $25.3 \%(\mathrm{n}=43)$ and $25.9 \%(\mathrm{n}=44)$ were readmitted to the hospital within 90 days after surgery. Conversion to open surgery, anastomotic leaks, and readmissions decreased over time.

Conclusions: MIILE can be performed safely and effectively with improving results as the surgeon's experience evolves. (J Thorac Cardiovasc Surg 2019;157:783-9)

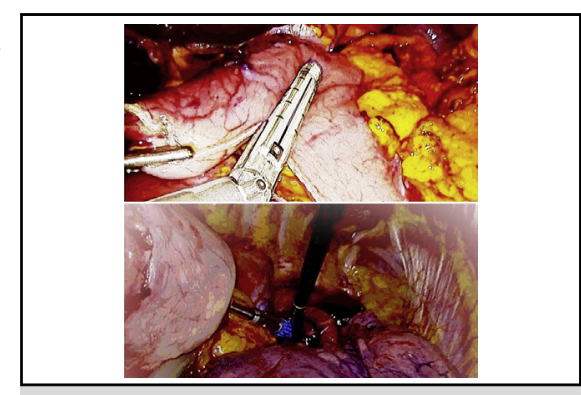

Creation of the conduit (above) and end-to-end anastomosis are key steps of the MIE.

\section{Central Message}

Minimally invasive Ivor Lewis esophagectomy is a safe and effective procedure for esophageal cancer, the results of which improve with the surgeon's experience.

\section{Perspective}

Minimally invasive Ivor Lewis esophagectomy is a technically demanding operation. In our study we evaluated its implementation relative to complications and readmission rates with graduated surgeon experience, and found that anastomotic leak and 90-day readmission rates were significantly different between early and late experience with the procedure.

See Editorial Commentary page 790.
The incidence of esophageal cancer has increased sixfold in the United States during the past 3 decades, and at present esophageal cancer is the sixth leading cause of death from

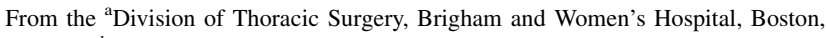
Mass; ${ }^{\mathrm{b}}$ Department of Biostatistics and Computational Biology, Dana-Farber Cancer Institute, Boston, Mass; and ' Thoracic Surgery Department, Xi' an Jiaotong University Medical College, First Affiliated Hospital, Xi'an, China.

Received for publication April 12, 2018; revisions received Sept 10, 2018; accepted for publication Oct 7, 2018; available ahead of print Nov 17, 2018.

Address for reprints: Abby White, DO, Division of Thoracic Surgery, Brigham and

Women's Hospital, 75 Francis St, Boston, MA 02115 (E-mail: Awhite12@bwh. harvard.edu).

$0022-5223 / \$ 36.00$

Copyright (c) 2018 by The American Association for Thoracic Surgery

https://doi.org/10.1016/j.jtcvs.2018.10.038
}

cancer worldwide. ${ }^{1}$ In 2017 alone, 16,940 new esophageal cancers were estimated to be diagnosed with an estimated 15,690 deaths in the United States. ${ }^{2}$

Surgery, as part of multimodality therapy, is the standard of care for locally advanced esophageal cancer. Nevertheless, esophagectomy has historically been associated with major in-hospital morbidity, readmission, and perioperative

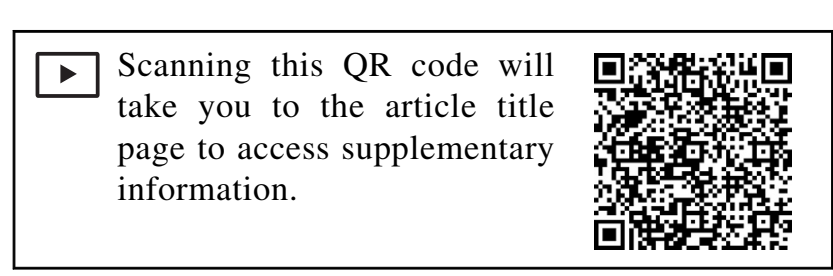




\section{Abbreviations and Acronyms \\ CI = confidence interval \\ $\mathrm{IQR}=$ interquartile range \\ MIILE $=$ minimally invasive Ivor Lewis esophagectomy}

mortality. Several innovations in the past 2 decades have enabled safer and less invasive esophageal surgery, and careful postoperative protocols in management have led to improved morbidity and mortality. ${ }^{3,4}$ Experience and available literature suggest that hospital readmissions after esophagectomy occur frequently, and are associated with worse survival. ${ }^{5}$ Thirty-day readmission rates for esophagectomy have been reported at $12 \%$ to $25 \%$, which exceeds the national average for all Medicare-billed admissions and surpasses those for most other cancer operations. Patients readmitted within 30 days of discharge after esophagectomy are at exceptionally high risk for early mortality. ${ }^{6,7}$ Although previous authors have shown safety and efficacy of minimally invasive Ivor Lewis esophagectomy (MIILE) ${ }^{8-10}$ it remains a technically demanding operation with several critical steps during laparoscopy and thoracoscopy. Little is published to inform individual thoracic surgeons and institutions about what to expect as experience with the operation and postoperative management expands. In this study we sought to assess the learning curve of MIILE and to evaluate perioperative outcomes, including anastomotic leak and hospital readmission, as a function of graduated surgeon experience.

\section{METHODS}

After institutional review board approval (2014P000998; April 16, 2018), a retrospective chart review was conducted for all patients who underwent minimally invasive esophagectomy by a single surgeon at the Brigham and Women's Hospital between April 2009 and April 2017. Data on patient demographic characteristics, history, and intraoperative and postoperative complications were extracted from the electronic medical record. Data regarding complications within 30 days and readmission (at any institution) within 90 days of surgery were collected.

Statistical analysis was performed using R (version 3.5.0) statistical software (https://www.R-project.org), as well as STATA 14 (StataCorp LP, College Station, Tex). We evaluated preliminarily the association between our main outcomes (the rate of readmission at 90 days from surgery, and the occurrence of 30-day complications) and a pool of covariates including: age at surgery, duration (in minutes) of surgery, smoking status (none, current smoker, former smoker), the forced expiratory volume in 1 minute (in percent), preoperative chemo- or radiotherapy, conversion to open surgery, Charlson comorbidity index score (1-9) the surgeon's experience in terms of number of performed surgeries, possible cofounders, such as deep vein thrombosis, pulmonary embolus, myocardial infarction, reintubation, aspiration, adult respiratory distress syndrome, pneumonia, empyema status, chylothorax, anastomotic leak, take back to operating room, and perioperative death, all codified with 0 or 1 according to whether or not each one occurred. For these comparisons, we used a univariable logistic regression.
TABLE 1. Multivariable logistic regression model for the 90-day readmission for patients who underwent MIILE

\begin{tabular}{lccc}
\hline \multicolumn{1}{c}{ Variable } & Odds ratio & $\mathbf{9 5} \%$ CI & $\boldsymbol{P}$ value \\
\hline $\begin{array}{l}\text { A. Final step of the selection method on the basis of the Akaike } \\
\text { information criterion }\end{array}$ & & & \\
Number of surgeries & 0.991 & $0.982-0.999$ & .026 \\
Age at surgery & 1.038 & $0.997-1.083$ & .080 \\
ARDS & N/A & N/A & - \\
Aspiration & N/A & N/A & - \\
DVT & 4.659 & $0.683-38.683$ & .114 \\
Pulmonary embolus & N/A & N/A & - \\
Empyema & 16.830 & $2.107-363.572$ & .019 \\
B. Final results of multivariate regression model (removing the \\
$\quad$ nonsignificant DVT covariate) & & \\
Number of surgeries & 0.991 & $0.983-0.998$ & .019 \\
Age at surgery & 1.044 & $1.006-1.086$ & .027 \\
Empyema & 6.814 & $1.141-56.648$ & .044 \\
\hline
\end{tabular}

$C I$, Confidence interval; $A R D S$, adult respiratory distress syndrome; N/A, not applicable, based on absence of events; $D V T$, deep vein thrombosis.

The results of the univariable analyses are reported in Tables 1 and 2. For many postoperative events, the odds ratios were either not defined (because of no events) or meaningless, because of a very low number of events (Table 2, section $A$ ). In these cases they have been omitted, and indicated with "N/A" in the corresponding tables.

We then analyzed the same data set from a multivariable perspective, using an adjusted logistic regression, with a forward stepwise variable selection method to determine the most significant predictors, on the basis of the value of the Akaike information criterion. The results for the significant covariates are reported in Tables 1 and 2. The "baseline," starting model contained only the intercept, whereas the "full" model contained all of the covariates listed previously. Chemo- or radiotherapy treatments were combined into a single binary covariate, describing whether or not the patients had received "any" treatment (between the two) or "no" treatment; also, we coded the surgeon's experience using an increasing, integer counter, representing the increasing, cumulative number of surgical procedures performed over time. Because it seemed unreasonable to assume a significant improvement in the surgical technique only because of the execution of a single esophagectomy, and because the data set is built in chronological order, we further subdivided the cumulative surgical experience into quartiles, to better highlight stages of possible improvement. Specifically, the first quartile included the first 44 MIILE operations performed by the surgeon at our institution and similarly, the fourth quartile included the most recent 41 operations performed by the same surgeon. Concurrently, we cross-tabulated each of the covariates listed previously with the binary covariate reporting whether the patients had had any complications after surgery, to estimate the occurrence rates. In general, differences in proportions were evaluated using the Fisher exact test, whereas the difference in medians (or means) with the Wilcoxon rank sum test.

We acknowledge the possible use of the cumulative sum control chart to detect change over time. However, we deemed that a simple univariable logistic regression (using only the predictor of the quartiles of the surgeon's experience) would be the most straightforward and easily interpretable choice to address the association of a multileveled variable (the quartiles) with a binary outcome (either the rate of readmission at 90 days and the rate of complications within 30 days).

The technical approach used is the MIILE, briefly summarized as follows. A 5-port laparoscopy is performed and gross metastatic disease ruled out. The greater curvature is mobilized, starting with the short gastric arteries. This is done with the Harmonic Scalpel (Ethicon, Cinncinati, Ohio). The lesser sac is entered and the hiatus is circumferentially dissected, identifying left and right crus. Any posterior attachments to 
TABLE 2. Results from different logistic models for the 30-day complication rate in patients who underwent MIILE

\begin{tabular}{|c|c|c|c|}
\hline Variable & Odds ratio & $95 \% \mathrm{CI}$ & $P$ value \\
\hline \multicolumn{4}{|c|}{ A. Covariates selected using the automatic forward selection procedure on the basis of the Akaike information criterion } \\
\hline Age at surgery & 1.020 & $0.980-1.052$ & .422 \\
\hline Reintubation & $4.61 \mathrm{E}+07$ & N/A & .993 \\
\hline Pneumonia & $1.63 \mathrm{E}+08$ & N/A & .995 \\
\hline DVT & $2.05 \mathrm{E}+08$ & N/A & .996 \\
\hline Anastomotic leak & $1.14 \mathrm{E}+08$ & N/A & .994 \\
\hline Chylothorax & $1.92 \mathrm{E}+08$ & N/A & .996 \\
\hline Aspiration & $1.68 \mathrm{E}+08$ & N/A & .996 \\
\hline \multicolumn{4}{|c|}{ B. Model excluding all "postoperative" covariates, likely to be highly collinear with the outcome (as shown in section A) } \\
\hline Number of surgeries & 0.994 & $0.985-1.002$ & .122 \\
\hline Age at surgery & 1.019 & 0.953-1.091 & .581 \\
\hline FEV1, \% & 0.996 & $0.979-1.013$ & 632 \\
\hline Surgery length, minutes & 1.002 & 0.996-1.007 & .554 \\
\hline \multicolumn{4}{|l|}{ Smoking status } \\
\hline Current & 2.199 & $0.657-7.772$ & .207 \\
\hline Former & 1.085 & $0.482-2.450$ & .843 \\
\hline Chemotherapy or radiation & 0.459 & $0.142-1.356$ & .170 \\
\hline \multicolumn{4}{|l|}{ Charlson Index } \\
\hline 1 & 1.017 & $0.184-6.050$ & .984 \\
\hline 2 & 1.781 & $0.235-14.319$ & .578 \\
\hline 3 & 1.489 & $0.201-11.591$ & 697 \\
\hline 4 & 0.930 & $0.093-9.445$ & .950 \\
\hline 5 & 1.695 & $0.109-26.744$ & .704 \\
\hline 6 & 4.767 & $0.260-104.166$ & .301 \\
\hline 7 & 1.065 & $0.019-54.997$ & .974 \\
\hline \multicolumn{4}{|c|}{ C. Multivariate logistic regression model only accounting for the most significant covariates from sections A and B } \\
\hline Number of surgeries & 0.994 & $0.987-1.000$ & .051 \\
\hline Age at surgery & 1.024 & $0.993-1.056$ & .138 \\
\hline
\end{tabular}

$C I$, Confidence interval; N/A, not applicable, due to 0 events; $D V T$, deep vein thrombosis; $F E V I$, forced expiratory volume in 1 minute.

the pancreas are lysed and the greater omentum mobilized from the greater curvature, taking care to avoid injury to the right gastroepiploic arcade. The left gastric artery is divided with a vascular endostapler. The pylorus should reach, tension free, to the hiatus at this point. A 4-cm wide gastric conduit is made with serial firings of the endostapler. This typically begins at the crow's feet on the lesser curvature and continues to just beyond the gastric fat pad. All lymph nodes in the gastric bed are removed particularly in the area of the celiac axis. A laparoscopic feeding jejunostomy is placed. Pyloroplasty is not routinely applied. The conduit is attached to the proximal specimen with 2 endosutures, carefully preserving the correct orientation. The posterior crural fibers are anchored with 1 endosuture to minimize the risk of a paraesophageal hernia. The patient is repositioned and a 5-port thoracoscopy performed. The esophagus is mobilized circumferentially. The azygous vein is typically divided, the specimen removed through the chest, and the anastomosis performed with an end-to-end anastomotic stapler; generally a $28-\mathrm{mm}$ anvil is used. The anastomosis is typically located from 23 to $25 \mathrm{~cm}$ from the incisors as documented by an esophagogastroduodenoscopy performed after the anastomosis is completed. All lymph nodes in the chest are removed from the periesophageal, subcarinal, and paratracheal spaces. A 10-French Jackson-Pratt drain is left in the mediastinum and a 24-French chest tube placed in the pleural space. Patients are extubated in the operating room. Video 1 shows a summary of the typical approach.

\section{RESULTS}

A total of 221 patients underwent esophagectomy by the same surgeon during the study period. Fifteen planned-open and 36 three-field esophagectomies were performed and these were excluded from the analysis. The remaining 170 patients who underwent MIILE were included in the analytic data set. The median age at surgery was

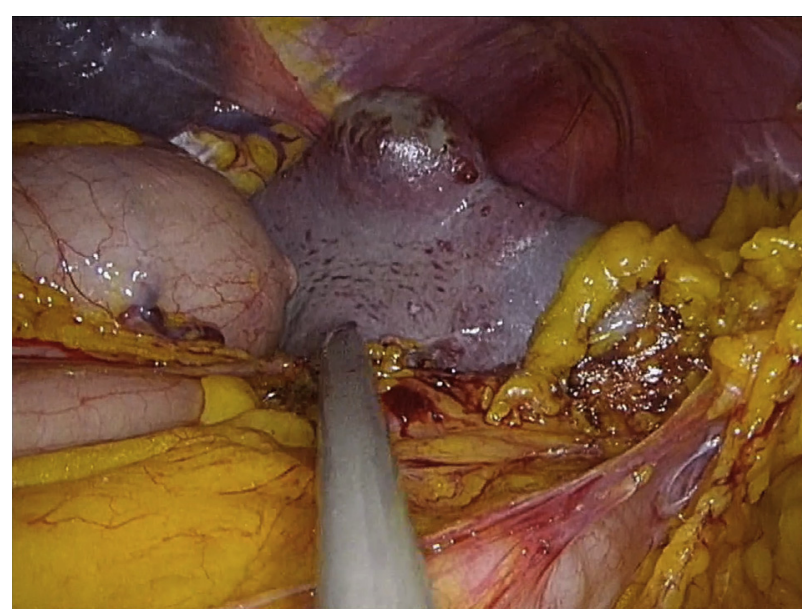

VIDEO 1. This video demonstrates the typical approach and technical steps applied for minimally invasive Ivor Lewis esophagectomy. Video available at: https://www.jtcvs.org/article/S0022-5223(18)32792-2/fulltext. 


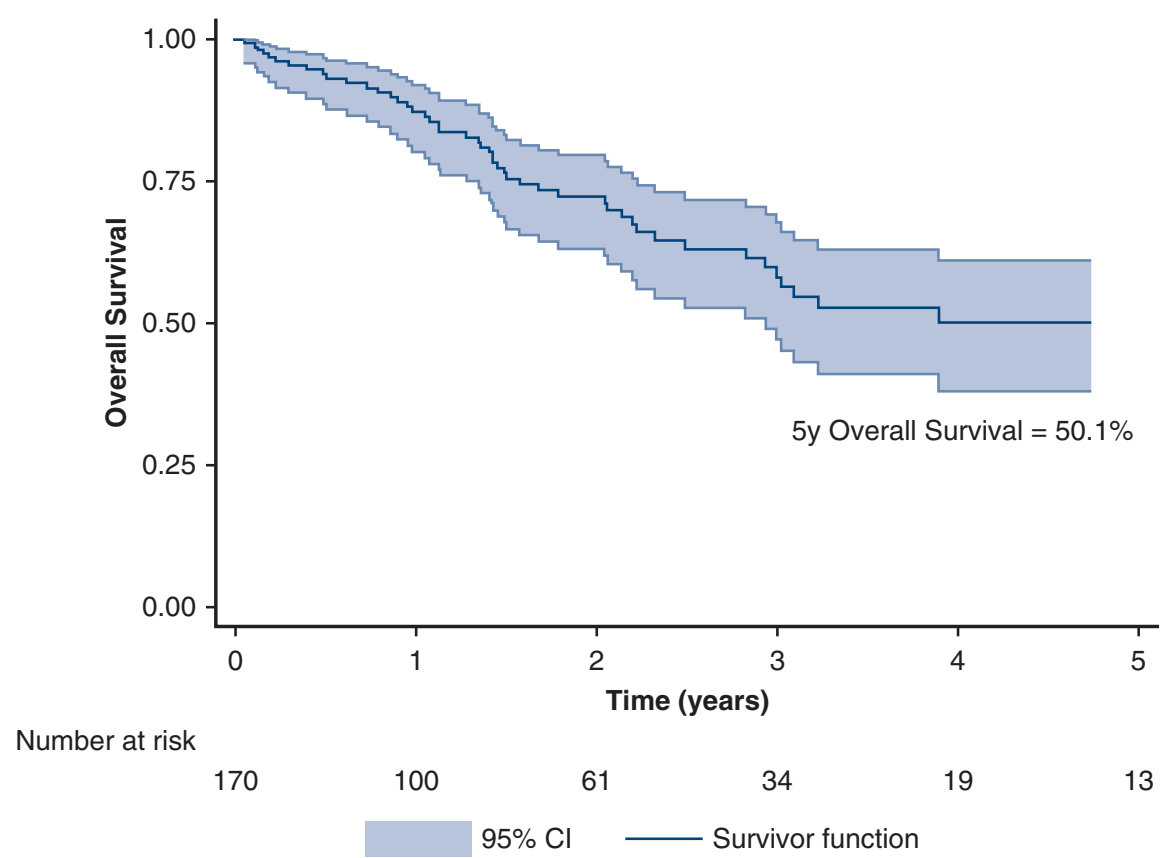

FIGURE 1. Kaplan-Meier survival estimates for patients who underwent MIILE for esophageal cancer $(\mathrm{n}=170)$. $C I$, Confidence interval.

64.7 years (range, 28.2-88.1 years; interquartile range [IQR], 58.4-72.0; mean, 64.6) and 147 patients (86.5\%) were male. The percent of patients who were either current or former smokers was 68.8. Adenocarcinoma was the most common histology $(94 \%)$. One hundred forty-three patients $(84.1 \%)$ received induction therapy, of whom 29 patients $(20 \%)$ showed a complete response on final pathologic review. The median operative time was 391 (range, 258-637; IQR, 350.5-444.0, with 11 missing values) minutes, and the median estimated blood loss was 250 (range, 50.0-2500.0 mL; IQR, 200.0-400.0; mean, 327.1) $\mathrm{mL}$. Three patients $(1.8 \%)$ received intraoperative blood transfusion. There were $8(4.7 \%)$ total conversions, in which either the chest or abdominal portion of the procedure was converted to open. The median postoperative length of stay was 8.0 (range, 6.0-64.0; IQR, 8.0-10.0; mean, 10.9) days. The 5-year overall survival for all patients was $50.1 \%$ (95\% confidence interval [CI], 39.7\%-63.2\%; Figure 1), whereas overall survival at 30 and 90 days was respectively $99.4 \%(95 \% \mathrm{CI}, 98.2 \%-100 \%)$ and $96.1 \%$ (95\% CI, 93.1\%-99.2\%).

Major complications occurred in $25.9 \%(n=43)$ and minor complications in $26.5 \%(\mathrm{n}=44)$. Anastomotic leak $(7.1 \%)$ and pneumonia $(4.7 \%)$ were the most common major complications. Anastomotic leaks were acknowledged by concerns of extraluminal contrast on postoperative upper gastrointestinal series, positive pleural fluid cultures, elevated pleural amylase, or changes in patient status that prompted further studies. All patients with concern for anastomotic leak, as detailed previously were, confirmed with esophagogastroduodenoscopy. The most common minor complication seen was atrial fibrillation $(24.1 \%)$. Forty-four patients $(25.9 \%)$ were readmitted within 90 days after surgery, including 13 patients who were readmitted more than once (5 patients twice, 6 three times, and 2 four times). The most common reason for readmission was dehydration, followed by pneumonia, fever, pleural effusion, and shortness of breath. Postoperative anastomotic stricture was defined as the need for 3 or more dilations within 6 months of esophagectomy and this occurred in 8 patients $(4.7 \%)$.

When considering the probability of readmission within 90 days from surgery as the outcome and using univariate logistic regressions to establish individual associations, we observed that the surgeon's experience, considered in this case as a one-by-one increasing indicator $(P=.01)$ and the age at surgery $(P=.04)$ were statistically significant factors for readmission. Also, specific complications were associated with a higher probability of being readmitted within 90 days of surgery: these included aspiration, empyema, anastomotic leak, and patients who experienced a return trip to the operating room. To evaluate the effect of surgeon experience on perioperative outcomes, we assumed that the effect of the surgeon's increased experience would be measurable if cumulated in quartiles. For this reason, we subdivided the integer counter in the data set representing the increasing surgeon's experience according to quartile (ie, subdividing all performed cases as much as possible into 4 equal subgroups), considering only the surgeon's experience as a covariate (Tables 3-5). 
TABLE 3. Relevant summaries of covariates of major interest stratified according to quartiles of surgeon's experience

\begin{tabular}{|c|c|c|c|c|c|c|}
\hline Quartile & Q1 & Q2 & Q3 & Q4 & All patients & $P$ value \\
\hline \multirow{3}{*}{$\begin{array}{c}\text { Number of } \\
\text { sequential } \\
\text { operations }\end{array}$} & $1-43$ & $44-85$ & $86-128$ & 129-170 & $1-170$ & \multirow{3}{*}{$\begin{array}{c}\text { Compares } \\
\text { earliest }(\mathrm{Q} 1) \\
\text { with most } \\
\text { recent }(\mathrm{Q} 4) \\
\text { experience }\end{array}$} \\
\hline & $\begin{array}{c}\text { Early } \\
\text { experience }\end{array}$ & & & $\begin{array}{c}\text { Recent } \\
\text { experience }\end{array}$ & & \\
\hline & $n=43$ & $n=42$ & $n=43$ & $n=42$ & $\mathbf{n}=\mathbf{1 7 0}$ & \\
\hline \multicolumn{7}{|l|}{ Mortality, $\%$} \\
\hline 30-Day & $0(0.0)$ & $1(2.4)$ & $0(0.0)$ & $0(0.0)$ & $1(0.6)$ & - \\
\hline 60-Day & $2(4.7)$ & $1(2.4)$ & $1(2.3)$ & $0(0.0)$ & $4(2.5)$ & .50 \\
\hline 90-Day & $2(4.7)$ & $3(7.3)$ & $1(2.3)$ & $0(0.0)$ & $6(3.9)$ & .50 \\
\hline 30-Day morbidity, n (\%) & $25(58.1)$ & $25(59.5)$ & $22(51.2)$ & $17(40.5)$ & $89(52.4)$ & .13 \\
\hline Anastomotic leak, n (\%) & $5(11.6)$ & $3(7.1)$ & $4(9.3)$ & $0(0.0)$ & $12(7.1)$ & .055 \\
\hline $\begin{array}{l}\text { Median time in OR } \\
\text { (IQR), minutes }\end{array}$ & $427.5(366.7-451.2)$ & $370.5(350.2-419.5)$ & $387.5(348.0-413.0)$ & $395.5(346.0-461.7)$ & $391.0(350.5-444.0)$ & .16 \\
\hline $\begin{array}{l}\text { Median length of stay } \\
(\text { IQR), d, n }\end{array}$ & $10(9-15)$ & $9(8-11.5)$ & $8(7.5-9)$ & $8(7-8.7)$ & $8(8-10)$ & $<.01$ \\
\hline $\begin{array}{l}\text { Median lymph nodes } \\
\text { sampled (IQR), n }\end{array}$ & $18(13-22.5)$ & $17.5(13-21.7)$ & $20(15.5-23.5)$ & $22(17-28.7)$ & $19(14-24)$ & .07 \\
\hline 90-Day readmission, n (\%) & $17(39.5)$ & $11(26.2)$ & $10(23.3)$ & $7(16.7)$ & $45(26.5)$ & .03 \\
\hline
\end{tabular}

Significant $P$ values are in bold. $Q 1-4$, Quartile 1-4; $O R$, operating room; $I Q R$, interquartile range.

Perioperative outcomes were recorded as a function of graduated surgeon experience within the 4 quartiles and as a single cohort (Table 3 ). There was no statistically significant difference between successive quartiles (ie, quartile [Q1] compared with Q2, Q2 compared with Q3) with respect to complications, morbidity, or mortality. However, a comparison of the cohort of patients representing the surgeons earliest experience (Q1) with that of the surgeons most recent experience revealed significant differences. The incidence of anastomotic leak was $11.6 \%$ early on (Q1) in the surgeon's experience, and decreased to 0 over time (Q4), a difference that approached statistical significance $(P=.055)$ using a Fisher exact test. Length of stay $(P<.001$, Wilcoxon rank sum test $)$ also decreased over time, as did readmission within 90 days of surgery $(P=.029$, Fisher exact test).

Patients who underwent surgery within the fourth quartile of experience had significantly lower overall odds of being readmitted within 90 days of their surgery compared with the first 43 patients who underwent the

TABLE 4. Univariable model for the 90-day readmission rate of patients who underwent MIILE, as a function of the single-surgeon experience in quartiles

\begin{tabular}{lcc}
\hline \multicolumn{1}{c}{ Quartile* $^{*}$} & Odds ratio $(\mathbf{9 5} \%$ CI $)$ & $\boldsymbol{P}$ value \\
\hline Q2 (44-85) & $0.599(0.233-1.499)$ & .277 \\
Q3 (86-128) & $0.511(0.195-1.293)$ & .162 \\
Q4 (129-170) & $0.337(0.115-0.908)$ & .037 \\
\hline
\end{tabular}

$C I$, Confidence interval; $Q 2-4$, quartile 2-4. *Quartiles compared with first experience Q1 containing cases 1-43. surgery (odds ratio, $0.34 ; P=.037$; Table 4). When we considered the rate of complications (any complication) after surgery, increasing surgeon experience showed an overall trend toward decreasing complications, although in this case, the effects were not statistically significant (Table 5). Smokers were 2.7 times as likely to experience a complication as a lifelong nonsmoker, though again this value was not statistically significant $(P=.08)$.

\section{DISCUSSION}

Esophagectomy is a complex and technically challenging surgical procedure, with many interdependent steps. Reduced morbidity, shorter hospital stays, and earlier return to function, among others, are well established benefits of minimally invasive surgery. The application of minimally invasive laparoscopic and thoracoscopic techniques to esophageal surgery has expanded the role of trimodality therapy and revolutionized perioperative care. The first thoracoscopic esophageal mobilizations for esophagectomy were performed in the early 1990s. ${ }^{11,12}$ After that, DePaula

TABLE 5. Univariable model for 30-day complication rate for patients who underwent MIILE, as a function of the single-surgeon experience in quartiles

\begin{tabular}{lcc}
\hline \multicolumn{1}{c}{ Quartile* } & Odds ratio $(\mathbf{9 5} \%$ CI $)$ & $\boldsymbol{P}$ value \\
\hline Q2 (44-85) & $1.059(0.445-2.525)$ & .897 \\
Q3 (86-128) & $0.754(0.320-1.765)$ & .516 \\
Q4 (129-170) & $0.490(0.203-1.153)$ & .105 \\
\hline
\end{tabular}

$\overline{C I \text {, Confidence interval; } Q 2-4 \text {, quartile 2-4. *Quartiles compared with first experience }}$ Q1 containing cases 1-43. 
and colleagues ${ }^{13}$ and Swanström and Hansen ${ }^{14}$ separately published the first totally minimally invasive transhiatal esophagectomies in 1995 and 1997, respectively. Subsequently, many centers began to adapt their favored technique to minimally invasive approaches and gained significant experience in laparoscopic and thoracoscopic approaches to esophagectomy, particularly led by Dr Luketich at Pittsburgh. ${ }^{15}$ Internationally, studies have evaluated the effect of "learning" minimally invasive esophagectomy. ${ }^{16,17}$ Nilsson and colleagues evaluated the staged implementation of a minimally invasive approach from open, to hybrid, to completely minimally invasive esophagectomy over a 4.5-year period. Less operative time, less blood loss, and fewer anastomotic leaks were noted compared with open surgery, however, statistical significance was not reported and the anastomotic leak rate with minimally invasive esophagectomy was reported to be as high as $15 \%$ to $29 \% .{ }^{16}$ A Dutch and Swedish study, which evaluated the learning curve among European surgeons with varying prestudy expertise, showed that anastomotic leak rates were as high as $18 \%$ initially, which decreased over time, although this did not reach statistical significance. This suggested considerable "learning-associated morbidity" and 119 was identified as the mean number of cases at which experience plateaued and "textbook" outcomes became more common. ${ }^{17}$ Interestingly though, the learning curve for individual surgeons varied considerably between surgeons. Despite available experience, little is known or understood about the individual learning curve for surgeons embracing minimally invasive esophagectomy in the United States. In the era of open surgery, it was shown that morbidity and mortality decreased with increasing surgeon and institution experience. ${ }^{18}$ As such, we hypothesized that there would be identifiable benefits of added experience in minimally invasive esophagectomy.

Readily observable is the reduction in anastomotic leak with increased surgeon experience. This is congruent with the observation that subtleties in technique, adapted over time as each patient informs the next, translated into appreciably fewer anastomotic issues. Although not statistically significant, the trend toward fewer overall complications with increasing surgeon experience is important, and shows the ability of individual surgeons to affect patient outcome in a paradigm of thoughtful investment, learning, and adapting. There was a reduction in readmissions within 90 days of operation as a function of surgeon experience. Increasing experience allowed us to anticipate specific issues, adapt technique, and adjust postoperative care algorithms. Finally, patients who experience aspiration, anastomotic leak, empyema, or a return trip to the operating room as part of the inhospital recovery are vulnerable to readmission and might benefit from closer surveillance after discharge.
Importantly, 7 of the total 8 conversions occurred in the first quartile, and only 1 in the fourth quartile, indicating increasing comfort with the technique and troubleshooting as time went on.

Our retrospective review is limited by sample size, but unique as a single-surgeon narrative. The reductions in readmissions and morbidities have been attributed to surgeon experience, however, general improvements in patient care over this time period might have affected these findings. These 2 covariates (year of treatment and surgeon's experience) might be collinear, so we deliberately noted (and assume for statistical purposes) that between 2009 and 2017 there have been no radical changes in perioperative care technique. Our pre-, intra-, and postoperative care strategies have remained largely the same. The inherent bias in technique and postoperative care provided by a single surgeon is part and parcel of the review. The consistency in surgical skill and judgement, however, is invaluable when assessing the learning curve of individual surgeons. Although not a template for training paradigms, we are obligated to ask how we prepare the next generation of minimally invasive surgeons to tackle the complexities of esophageal surgery. Our study suggests we can expect comparable oncologic and safety profiles early on, and that these will get even better over time. MIILE is associated with improved perioperative outcomes without compromising survival. Our study supports the ongoing adoption of minimally invasive technologies in the surgical management of esophageal cancer.

\section{CONCLUSIONS}

MIILE is safe and effective for esophageal cancer, the results of which improve with the surgeon's experience.

\section{Conflict of Interest Statement}

Dr Swanson is a consultant for Covidien and Ethicon. All other authors have nothing to disclose with regard to commercial support.

\section{References}

1. Pohl H, Welch HG. The role of over diagnosis and reclassification in the marked increase of esophageal adenocarcinoma incidence. J Natl Cancer Inst. 2005;97: $142-6$.

2. American Cancer Society. Estimated number of new cancer cases and deaths by sex, US, 2017. Available at: https://www.cancer.org/research/cancer-factsstatistics/all-cancer-facts-figures/cancer-facts-figures-2017.html. Accessed May 26, 2017.

3. Wee JO, Bueno R, Swanson SJ. Minimally invasive esophagectomy: the Brigham and Women's Hospital experience. Ann Cardiothorac Surg. 2017;6:175-8.

4. Spector R, Zheng Y, Yeap BY, Wee JO, Lebenthal A, Swanson SJ, et al. The 3-hole minimally invasive esophagectomy: a safe procedure following neoadjuvant chemotherapy and radiation. Semin Thorac Cardiovasc Surg. 2015;27:205-15.

5. Fernandez FG, Khullar O, Force SD, Jiang R, Pickens A, Howard D, et al. Hospital readmission is associated with poor survival after esophagectomy for esophageal cancer. Ann Thorac Surg. 2015;99:292-7. 
6. Elixhauser A, Steiner C. Readmissions to US hospitals by diagnosis, 2010: Statistical brief \#153. Healthcare cost and utilization project (HCUP) statistical briefs. Rockville, Md: Agency for Healthcare Research and Quality; 2006.

7. Hu Y, McMurry TL, Stukenborg GJ, Kozower BD. Readmission predicts 90-day mortality after esophagectomy: analysis of Surveillance, Epidemiology, and End Results registry linked to Medicare outcomes. J Thorac Cardiovasc Surg. 2015; 150:1254-60

8. Wee JO. Minimally invasive oesophagectomy: the Ivor Lewis approach. Multimed Man Cardiothorac Surg. 2015;2015:mmv034.

9. Luketich JD, Pennathur A, Awais O, Levy RM, Keeley S, Shende M, et al, Outcomes after minimally invasive esophagectomy: review of over 1000 patients. Ann Surg. 2012;256:95-103.

10. Luketich JD, Pennathur A, Franchetti Y, Catalano PJ, Swanson S, Sugarbaker DJ, et al. Minimally invasive esophagectomy: results of a prospective phase II multicenter trial-the Eastern Cooperative Oncology Group (E2202) study. Ann Surg. 2015;261:702-7.

11. Cuschieri A, Shimi S, Banting S. Endoscopic oesophagectomy through a right thoracoscopic approach. J R Coll Surg Edinb. 1992;37:7-11.

12. Collard JM, Lengele B, Otte JB, Kestens PJ. En bloc and standard esophagectomies by thoracoscopy. Ann Thorac Surg. 1993;56:675-9.
13. DePaula AL, Hashiba K, Ferreira EA, de Paula RA, Grecco E. Laparoscopic transhiatal esophagectomy with esophagogastroplasty. Surg Laparosc Endosc. $1995 ; 5: 1-5$.

14. Swanström LL, Hansen P. Laparoscopic total esophagectomy. Arch Surg. 1997 132:943-7; discussion: 47-9.

15. Luketich JD, Alvelo-Rivera M, Buenaventura PO, Christie NA, McCaughan JS, Litle VR, et al. Minimally invasive esophagectomy: outcomes in 222 patients. Ann Surg. 2003;238:486-94; discussion: 94-5.

16. Nilsson M, Kamiya S, Lindblad M, Rouvelas I. Implementation of minimally invasive esophagectomy in a tertiary referral center for esophageal cancer J Thorac Dis. 2017;9:S817-25.

17. Workum F, Stestra MH, Berkelmans GH, Slaman AE, van Berge Henegouwen MI, Gisbertz SS, et al. Learning curve and associated morbidity of minimally invasive esophagectomy. Ann Surg. 2019;269:88-94.

18. Orringer MB, Marshall B, Chang AC, Lee J, Pickens A, Lau CL. Two thousand transhiatal esophagectomies: changing trends, lessons learned. Ann Surg. 2007; 246:363-72; discussion: 372-4.

Key Words: esophageal cancer, minimally invasive surgery 